\title{
A non-stationary relay-based 3D MIMO channel model with time-variant path gains for human activity recognition in indoor environments
}

\author{
Rym Hicheri $^{1}$ (D) Ahmed Abdelgawwad ${ }^{1} \cdot$ Mathias Pätzold $^{1}$
}

Received: 20 July 2020 / Accepted: 26 March 2021 / Published online: 14 May 2021

(C) The Author(s) 2021

\begin{abstract}
Extensive research showed that the physiological response of human tissue to exposure to low-frequency electromagnetic fields is the induction of an electric current in the body segments. As a result, each segment of the human body behaves as a relay, which retransmits the radio-frequency (RF) signal. To investigate the impact of this phenomenon on the Doppler characteristics of the received RF signal, we introduce a new three-dimensional (3D) non-stationary channel model to describe the propagation phenomenon taking place in an indoor environment. Here, the indoor space is equipped with a multiple-input multiple-output (MIMO) system. A single person is moving in the indoor space and is modelled by a cluster of synchronized moving point scatterers, which behave as relays. We derive the time-variant (TV) channel transfer function (CTF) with TV path gains and TV path delays. The expression of the TV path gains is obtained from the instantaneous total received power at the receiver side. This TV total received power is expressed as the product of the TV power of the RF signal initially transmitted and received by a body segment and the TV received power of the redirected signal. These TV powers are determined according the free-space path-loss model. Also, a closed-form approximate solution to the spectrogram of the TVCTF is derived. Here, we analyse the effect of the motion of the person and the validity of the relay assumption on the spectrogram, the TV mean Doppler shift (MDS), and the TV Doppler shift (DS) of the TVCTF. Simulation results are presented to illustrate the proposed channel model.
\end{abstract}

Keywords Non-stationary channels $\cdot$ Doppler characteristics $\cdot$ Time-variant path gains $\cdot$ Spectrogram $\cdot$ Multiple-input multiple-output systems $\cdot$ Human activities recognition

\section{Introduction}

According to the World Health Organization (WHO), the elderly population (ages $65+$ ) is expected to triple in the

This paper was presented in parts at the International Conference on Telecommunications and Computing Technologies (ICTCT'20), Hammamet, Tunisia, November 2020 [13].

\section{Rym Hicheri \\ rym.hicheri@uia.no}

Ahmed Abdelgawwad

ahmed.abdel-gawwad@uia.no

Ahmed Abdelgawwad

matthias.paetzold@uia.no

1 Faculty of Engineering and Science, University of Agder, NO-4898 Grimstad, Norway next 30 years and reach $16 \%$ of the global population [2]. Thus, there has been a great focus on the study of home care systems based on human activity recognition, tracking, and classification techniques. This is of great interest as most older people prefer to live independently in their own homes [10]. But, living alone, albeit in a familiar environment, carries the risk of incidents like falls and unconsciousness [31]. For example, as reported in [34], approximately $30 \%$ of people over 65 and $50 \%$ of people over 85 fall at least once a year. This calls for robust in-home tracking and monitoring systems.

A review of the literature shows that human activity recognition systems utilize three major techniques: video or optical marker-based surveillance, wearable and contextaware inertial measurement units (IMUs), and radio frequency (RF) signal processing [14, 16, 21]. With the recent developments in computer vision and digital cameras, vision-based and/or marker-based techniques have 
been widely investigated in the context of in-home activity monitoring. Here, the markers are placed on the human body and must be clearly visible to the cameras. They are based on extensive image processing and/or the use of optical marker-based motion capture systems, e.g., [15, 17, 25]. Wearable and context-aware systems use IMUs which can be placed on the person (such as watch, smartphone, clothes, etc.) or deployed in the room (along the floor or on the walls). They employ the embedded accelerometers, gyroscopes, and magnetometers data to identify and classify human activities [24, 28]. Apart from privacy concerns, the main drawbacks of the first two categories are that the person may forget to wear the sensors-based devices or move outside the detection range of the non-wearable sensors/cameras [30]. This is of practical importance, especially for the elderly population. Non-wearable RFbased systems have been introduced as a solution to overcome the disadvantages of the aforementioned systems [21]. These RF-based systems track and monitor indoor human activities by analyzing and exploiting the effects of the human motion on the Doppler characteristics of the transmitted RF signals. Several parameters can be utilized for this underlying task such as the received signal strength, the TV phase of the received signal, the time-of-arrival, the time differences of arrival, and the angle-of-arrival. More papers on this topic can be found in $[7,12,18,20,23,32$, 33].

The design, development, and implementation of efficient RF-based methods rely heavily on accurate indoor channel models to describe the non-stationary multipath propagation phenomenon. The majority of the aforementioned studies are based on scenario specific multipath channel models, where the moving person or object is modelled by a single point scatterer, and assume constant path gains when expressing the received RF signal. A fixed-to-fixed (F2F) single-input single-output (SISO) three-dimensional (3D) channel model with constant path gains has been reported in [3], where the body segments of the moving person have been modelled by a cluster of synchronized moving point scatterers. The authors analyzed the effects of the motion of the person on the spectrogram of the complex channel gain, the TV mean Doppler shift (MDS), and TV Doppler spread (DS). More recently, a F2F SISO 3D nonstationary channel model with a stochastic trajectory model has been presented in [6], where the person is modelled by a single moving scatterer. The expression of the complex channel gain of this model incorporates TV path gains that change with the total distance travelled by the moving person from the transmitter to the receiver. In [6], the TV path gains are determined according to the path loss model [29] by considering the sum of the TV distances between the body segments (moving scatterers) and the transmitter/receiver. In other words, diffuse reflection is assumed.
This means that the incoming radio waves bounce back in all directions without absorption, redirection, or any physical alteration. This assumption is not consistent with the results of the studies reported in $[1,8,9,22]$, in which the effects of electromagnetic fields, particularly in the extreme low-frequency range, on the human body were investigated. As shown in $[1,8,9,22]$, when exposed to a low-frequency electromagnetic field, the human tissues (body) generate(s) an electric current. This current is generally referred to as "induced current." In this case, the human body does not reflect the incoming radio waves, but it retransmits them. Thus, the different body segments of the moving person (moving point scatterers) are assumed to play the role of relays, which redirect the transmitted RF signal. This problem was initially addressed in [13], where a new channel model with TV path gains and TV path delays was introduced. In [13], each body segment is assumed to play the role of a moving relay. In this case, the TV path gain of each multipath component is computed in terms of the product of the mean power of the initially transmitted signal and the mean power of the redirected signal (received by the receive antennas). These mean powers can be modelled according to the free-space path loss model [29]. Here, the result depends on the product of the TV distance between the transmit antenna and the moving scatterer (body segment) and the TV distance between the same moving scatterer and the receive antenna. This problem was initially studied in [13]. In [13], relay properties are applied to compute the total received power, from which the expression of the TV path gains is derived.

With this paper, we extend the work in [13] to address this problem. To do so, we present a new generic F2F multiple-input multiple-output (MIMO) 3D channel model to describe the non-stationary behavior of indoor environments. Here, the different body segments of the moving person (modelled by a synchronized cluster of moving point scatterers) play the role of moving relays. The impacts of the fixed objects and the line-of-sight component (LOS) are taken into account. Since they do not experience Doppler effects, they can be modelled by fixed point scatterers with constant path gains and constant path delays. Here, we present an expression for the TV channel transfer function (CTF) of the received RF signal with TV path gains and TV path delays. The free-space path loss model [29] is applied to describe the total received power of the initially transmitted signal and the received power of the redirected radio signal. Later, a closed-form approximate solution to the spectrogram of the TVCTF is derived. The influence of introducing TV path gains and assuming the relay-like behavior of the different body segments on the Doppler characteristics of the channel is analyzed. The impact of considering TV path gains and multiple antenna elements at both the transmitter side and receiver side is 
also investigated. These tasks are achieved by means of the spectrogram of the TVCTF, the TV mean Doppler shift (MDS), and the TV Doppler spread (DS). Simulation results are presented to illustrate the proposed channel model.

The remaining of this paper is organised as follows. Section 2 discusses the indoor propagation scenario, where the person is modelled by a cluster of synchronized moving scatterers. Then, the total power of the received RF signal is determined in Section 3. The derivation of the TVCTF of the received RF signals is presented in Section 4 together with the corresponding spectrogram. Section 5 presents some numerical results and Section 6 concludes the paper.

\section{Scenario description}

As illustrated in Fig. 1, we consider an example of a typical rectangular cuboid room. The room of length $A$, width $B$, and height $H$, is equipped with an $N_{T} \times N_{R}$ MIMO communication system. Here, $N_{T}$ denotes the number of transmit antennas $A_{j}^{T}\left(j=1, \ldots, N_{T}\right)$ and $N_{R}$ designates the number of receive antennas $A_{i}^{R}\left(i=1, \ldots, N_{R}\right)$. There are several stationary (fixed) objects (e.g., walls, furniture, and decoration items) and a single moving person. The moving person is modelled by a cluster of moving scatterers, where each single point scatterer $(\star)$ represents a segment of the human body such as head, shoulders, arms, etc.

The corresponding geometrical 3D channel model shown in Fig. 2 is the starting point for modelling the indoor propagation phenomenon. Each transmit (receive) antenna element $A_{j}^{T}\left(A_{i}^{R}\right)$ is located at the fixed coordinates $\left(x_{j}^{T}, y_{j}^{T}, z_{j}^{T}\right)\left(\left(x_{i}^{R}, y_{i}^{R}, z_{i}^{R}\right)\right), j=1,2, \ldots, N_{T} \quad(i=$ $\left.1,2, \ldots, N_{R}\right)$. Here, we assume the presence of LOS components.

The fixed objects, which can be seen between the antennas $A_{i}^{T}$ and $A_{j}^{R}$, are modelled by $K_{i j}$ fixed point

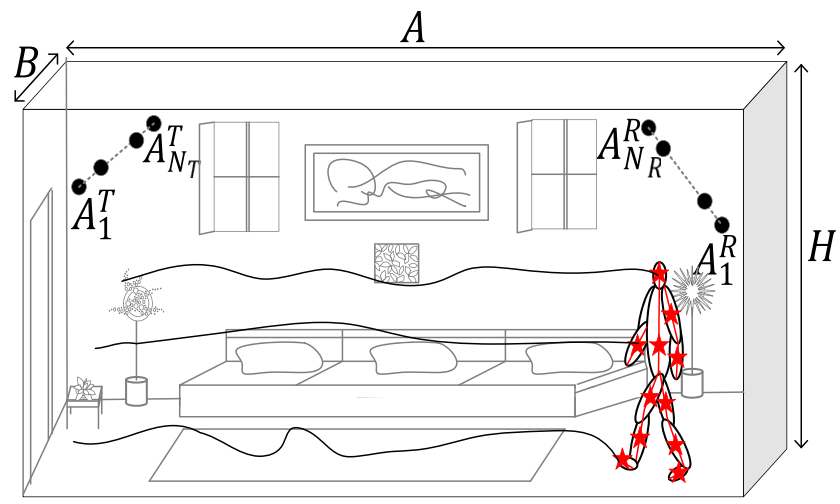

Fig. 1 A single moving person (modelled by a cluster of moving scatterers) walks in a room equipped with a MIMO system and several fixed (stationary) objects [13]

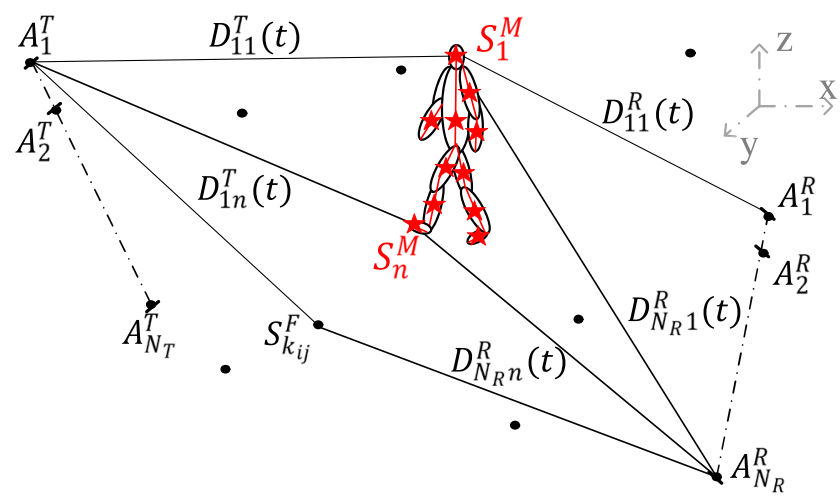

Fig. 2 The 3D geometrical model for an $N_{T} \times N_{R}$ MIMO channel with fixed $(\bullet)$ and moving scatterers $(\star)$ [13]

scatterers $(\bullet)$. These fixed scatterers are denoted by $S_{k_{i j}}^{F}$, $k_{i j}=1,2, \ldots, K_{i j}$. On the other hand, every body segment of the moving person is modelled by a single moving point scatterer $(\star)$, denoted by $S_{n}^{M}(n=1,2, \ldots, M)$, which is located at the TV position $\left(x_{n}(t), y_{n}(t), z_{n}(t)\right)$. Singlebounce scattering is assumed when modelling the fixed and moving scatterers.

\section{Total received power}

Together with considering TV path gains and TV path delays, the other novelty of this work is that the human body segments are assumed to play the role of relays, which redirect the transmitted radio wave. This is motivated by the fact that low-frequency electromagnetic fields have been shown to induce (in)perceivable electric currents in the human body. In turn, these "induced" currents can cause effects that vary from a dermal reaction to a tingling sensation depending on the frequency range [1, 8, 9, 22]. In other words, and as illustrated in Fig. 3, when the incoming (transmitted) RF signal (_) impinges on a body segment (moving scatterer $(\star)$ ), an electric current is induced $\left(_{-}\right)$ that retransmits the RF signal in all directions (_). In the presence of these induced currents (_), the human body segments (moving scatterers) play the role of relays.

As can be seen in Fig. 3, the propagation phenomenon can be divided into three phases. In phase I, the RF signal is transmitted from the antenna element $A_{j}^{T}$ and received by the $n$th body segment $S_{n}^{M}$ with an instantaneous power $P_{j n}^{T}(t)$. In phase II, the electric current is induced, and the power of the received signal is amplified by a factor $a_{n}^{2}$, which describes the contribution of $S_{n}^{M}$ with respect to the entire human body and satisfies the condition $\sum_{n=1}^{M} a_{n}^{2}=1$. In relay systems, the quantity $a_{n}^{2}$ is commonly referred to as the "cross-section" parameter. Finally, in phase III, the 


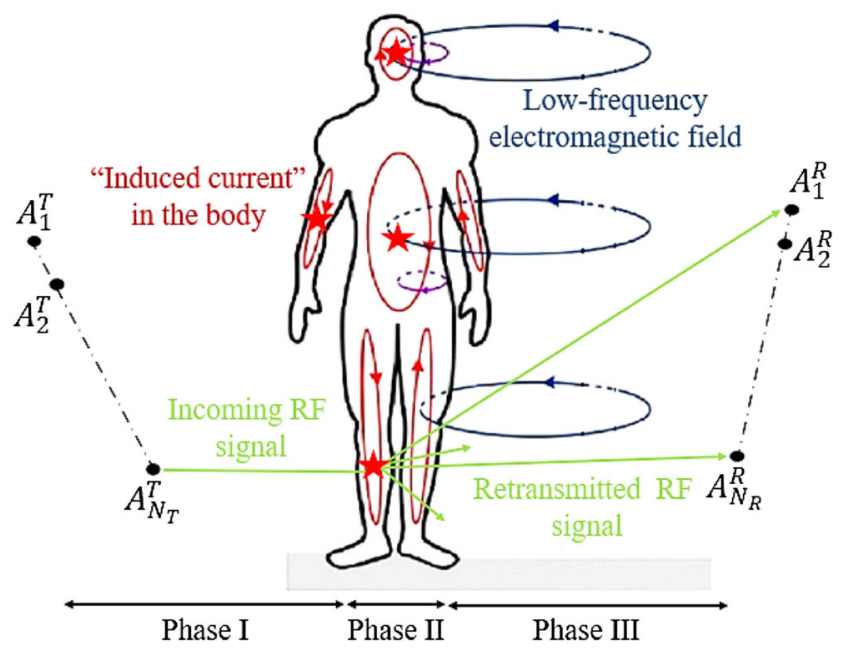

Fig. 3 Behavior of human body segments in the presence of lowfrequency electromagnetic fields

signal is retransmitted from the scatterer $S_{n}^{M}$ and received at the receive antenna $A_{i}^{R}$ with an instantaneous power $P_{i n}^{R}(t)$.

We denote by $P_{i j n}(t)$ the total instantaneous received power of the RF signal transmitted from the $j$ th transmit antenna $A_{j}^{T}$ and received at the $i$ th receive antenna $A_{i}^{R}$ after being relayed by the $n$th moving scatterer $S_{n}^{M}$. Assuming that the body segments play the role of relays, the total TV received power $P_{i j n}(t)$ can be expressed as the product of the TV powers $P_{j n}^{T}(t)$ and $P_{i n}^{R}(t)$ and the cross-section parameter $a_{n}^{2}$, i.e.,

$P_{i j n}(t)=P_{j n}^{T}(t) \times a_{n}^{2} \times P_{i n}^{R}(t)$.

The wave propagation phenomena in phases I and III are taking place in a free-space environment. Then, the freespace path loss model $[11,29]$ can be employed to express the TV received powers $P_{j n}^{T}(t)$ and $P_{i n}^{R}(t)$ as $P_{i n}^{R}(t)=$ $C\left[D_{j n}^{T}(t)\right]^{-\gamma}$ and $P_{i n}^{R}(t)=C\left[D_{i n}^{R}(t)\right]^{-\gamma}$, respectively. Here, the quantity $C$ depends on the transmit/receive antenna gain, the transmission power, and the wavelength [29], while $\gamma$ is the path loss exponent which is equal to 2 in free space and between 1.6 and 1.8 in indoor environments. Moreover, the quantity $D_{j n}^{T}(t)\left(D_{i n}^{R}(t)\right)$ represents the TV distance between the $j$ th ( $i$ th) transmit (receive) antenna $A_{j}^{T}$ $\left(A_{i}^{R}\right)$ and the $n$th moving scatterer $S_{n}^{M}, n=1,2, \ldots, M$. According to Fig. 2, the TV distances $D_{j n}^{T}(t)$ and $D_{i n}^{R}(t)$ can be expressed as

$D_{j n}^{T}(t)=\left[\left(x_{n}(t)-x_{j}^{T}\right)^{2}+\left(y_{n}(t)-y_{j}^{T}\right)^{2}+\left(z_{n}(t)-z_{j}^{T}\right)^{2}\right]^{\frac{1}{2}}(2)$

and

$D_{i n}^{R}(t)=\left[\left(x_{i}^{R}-x_{n}(t)\right)^{2}+\left(y_{i}^{R}-y_{n}(t)\right)^{2}+\left(z_{i}^{R}-z_{n}(t)\right)^{2}\right]^{\frac{1}{2}}$ respectively. Finally, inserting the expressions of $P_{j n}^{T}(t)$ and $P_{i n}^{R}(t)$ in Eq. 1 yields the following expression for the instantaneous received power $P_{i j n}(t)$

$P_{i n}^{R}(t)=a_{n}^{2} C^{2}\left[D_{i n}^{R}(t) \times D_{j n}(t)^{T}\right]^{-\gamma}$.

The total instantaneous power $P_{i j n}(t)$ represents the power of the signal transmitted by the $j$ th transmit antenna $A_{j}^{T}$ and received at the $i$ th receive antenna $A_{i}^{R}$ after being relayed by the $n$th moving scatterer (body segment) $S_{n}^{M}$.

In the following section, the result in Eq. 4 will be employed to express the TV path gains of the moving scatterers.

\section{3D non-stationary channel model}

According to Fig. 2, the TVCTF $H_{i j}\left(f^{\prime}, t\right)$ of the subchannel between the $j$ th transmit antenna $A_{j}^{T}$ and the $i$ th receive antenna $A_{i}^{R}$ can be expressed as

$$
\begin{aligned}
H_{i j}\left(f^{\prime}, t\right)= & \sum_{n=1}^{M} c_{i j n}(t) \exp \left(j\left(\theta_{i j n}-2 \pi\left(f_{c}+f^{\prime}\right) \tau_{i j n}(t)\right)\right) \\
& +\sum_{k=0}^{K_{i j}} c_{k_{i j}} \exp \left(j\left(\theta_{k_{i j}}-2 \pi\left(f_{c}+f^{\prime}\right) \tau_{k_{i j}}\right)\right)
\end{aligned}
$$

where $f_{c}$ is the center frequency and $t\left(f^{\prime}\right)$ refers to the time (frequency) domain.

The first part of Eq. 5 describes the effects introduced by the moving point scatterers (modelling the centres of mass of the major segments of the human body). Here, the quantities $c_{i j n}(t)$ and $\tau_{i j n}(t)$ are the TV path gain and TV path delay of the $n$th moving scatterer $S_{n}^{M}(n=1,2, \ldots, M)$, respectively. These parameters are given by

$c_{i j n}(t)=\sqrt{P_{i n}^{R}}=a_{n} C\left[D_{i n}^{R}(t) \times D_{j n}^{T}(t)\right]^{-\gamma / 2}$

and

$\tau_{i j n}(t)=\frac{D_{j n}^{T}(t)+D_{i n}^{R}(t)}{c_{0}}$

respectively, where the quantity $c_{0}$ denotes the speed of light. It is worth mentioning that the instantaneous Doppler frequencies $f_{i j n}\left(t, f^{\prime}\right)$ of the $n$th moving scatterer $S_{n}^{M}$ ( $n=1,2, \ldots, M)$ can be obtained from the TV delays $\tau_{i j n}(t)$ according to the fundamental relationship $f_{i j n}(t)=$ $-\left(f_{c}+f^{\prime}\right) \tau_{i j n}(t)$ [27]. In the Appendix, mathematical details are presented showing that for short time intervals $\left[t_{0}-\delta t / 2, t_{0}+\delta t / 2\right]$ centered at the time instant $t_{0}$ and of duration $\delta t$, the TV path gains $c_{i j n}(t)$ can be assumed constant and expressed according to Eq. 24. The initial phases of the channel, denoted by $\theta_{i j n}\left(j=1,2, \ldots, N_{T}\right.$, $i=1,2, \ldots, N_{R}$, and $\left.n=1,2, \ldots, M\right)$, are modelled 
by independent and uniformly distributed (i.i.d.) random variables which are uniformly distributed over the interval $[0,2 \pi)$.

The second sum of Eq. 5 models the multipath propagation effect resulting from the fixed scatterers (objects) $S_{k_{i j}}^{F}, k_{i j}=1,2, \ldots, K_{i j}$. Each fixed scatterer $S_{k_{i j}}$ is described by a constant path gain $c_{k_{i j}}$, a constant path delay $\tau_{k_{i j}}^{\prime}$, and a constant phase $\theta_{k_{i j}}, k=1,2, \ldots, K_{i j}$.

The LOS component, which does not experience any Doppler effects, can be modelled by a fixed scatterer $S_{0}^{F}$ $\left(k_{i j}=0\right)$, with a constant path gain $c_{0_{i j}}$ and a constant delay $\tau_{0_{i j}}$. The phases $\theta_{k_{i j}}$ are modelled by i.i.d. random variables with a uniform distribution over the interval $[0,2 \pi)$.

The aim of the present work is to investigate the impact of the TV path gains $c_{i j n}(t)$ on the channel characteristics independently of the individual contributions of the fixed scatterers $S_{k_{i j}}$. Therefore, for simplicity, the overall effect of the $K_{i j}$ fixed scatterers, i.e., the second sum of Eq. 5, is replaced by a single complex term $c_{i j F}\left(f^{\prime}\right) \exp \left[j\left(\vartheta_{i j F}\left(f^{\prime}\right)\right)\right]$, with a magnitude $c_{i j F}\left(f^{\prime}\right)$ and an argument $\vartheta_{i j F}\left(f^{\prime}\right)$. The quantities $c_{i j F}\left(f^{\prime}\right)$ and $\vartheta_{i j F}\left(f^{\prime}\right), j=1,2, \ldots, N_{T}$, and $i=1,2, \ldots, N_{R}$, are expressed in terms of the fixed path gains $c_{k_{i j}}$, phases $\theta_{k_{i j}}$, and path delays $\tau_{k_{i j}}$ as

$$
\begin{aligned}
c_{i j F}\left(f^{\prime}\right)= & \left\{\left[\sum_{k=0}^{K_{i j}} c_{k_{i j}} \cos \left(\theta_{k_{i j}}-2 \pi f^{\prime} \tau_{k_{i j}}^{\prime}\right)\right]^{2}\right. \\
& \left.+\left[\sum_{k=0}^{K_{i j}} c_{k_{i j}} \sin \left(\theta_{k_{i j}}-2 \pi f^{\prime} \tau_{k_{i j}}^{\prime}\right)\right]^{2}\right\}^{1 / 2}
\end{aligned}
$$

and

$$
\begin{aligned}
\vartheta_{i j F}\left(f^{\prime}\right)= & \operatorname{atan} 2\left(\sum_{k=0}^{K_{i j}} c_{k_{i j}} \sin \left(\theta_{k_{i j}}-2 \pi f^{\prime} \tau_{k_{i j}}^{\prime}\right),\right. \\
& \left.\sum_{k=0}^{K_{i j}} c_{k_{i j}} \cos \left(\theta_{k_{i j}}-2 \pi f^{\prime} \tau_{k_{i j}}^{\prime}\right)\right)
\end{aligned}
$$

$$
\begin{aligned}
S_{i j}^{(c)}\left(f^{\prime}, f, t\right)= & \frac{2}{\sigma_{w} \pi}\left[\sum _ { n = 1 } ^ { M - 1 } \sum _ { l = n + 1 } ^ { M } c _ { i j n } ( t ) c _ { i j l } ( t ) \operatorname { R e } \left\{G\left(f,-\left(f_{c}+f^{\prime}\right) \dot{\tau}_{n}(t), \sigma_{i j n, 2}^{2}\left(f^{\prime}, t\right)\right) G^{*}\left(f,-\left(f_{c}+f^{\prime}\right) \dot{\tau}_{l}(t), \sigma_{i j l, 2}^{2}\left(f^{\prime}, t\right)\right)\right.\right. \\
& \left.\cdot \exp \left(j\left(\theta_{i j n}-\theta_{i j l}-2 \pi\left(f_{c}+f^{\prime}\right)\left(\tau_{i j n}(t)-\tau_{i j l}(t)\right)\right)\right)\right\}+\sum_{n=1}^{M} c_{i j n}(t) c_{i j F}\left(f^{\prime}\right) G\left(f, 0, \sigma_{F}^{2}\right) \\
& \left.\cdot \operatorname{Re}\left\{G\left(f,-\left(f_{c}+f^{\prime}\right) \dot{\tau}_{n}(t), \sigma_{i j n, 2}^{2}\left(f^{\prime}, t\right)\right) \exp \left(j\left(\theta_{i j n}-\vartheta_{i j F}\left(f^{\prime}\right)-2 \pi\left(f_{c}+f^{\prime}\right) \tau_{i j n}(t)\right)\right)\right\}\right]
\end{aligned}
$$

respectively, where atan2(.) is the inverse tangent function and returns a value in the interval $[-\pi, \pi[[26]$.

To visualize the influence of introducing TV path gains to describe the motion of the person (modelled by a cluster of synchronized moving scatterers), we consider the spectrogram $S_{i j}\left(f^{\prime}, f, t\right)$ of the TVCTF $H_{i j}\left(f^{\prime}, t\right)$ using a Gaussian window $[3,6]$.

According to [3], the spectrogram $S_{i j}\left(f^{\prime}, f, t\right)$ of the TVCTF $H_{i j}\left(f^{\prime}, t\right)$ is given by the absolute square of the shorttime Fourier transform $X_{i j}\left(f^{\prime}, f, t\right)$ of the product of the TVCTF $H_{i j}\left(f^{\prime}, t\right)$ and a sliding Gaussian window $h(t)$ of spread $\sigma_{w}$. Therefore, the spectrogram $S_{i j}\left(f^{\prime}, f, t\right)$ of the TVCTF $H_{i j}\left(f^{\prime}, t\right)$ can be expressed as

$$
\begin{aligned}
S_{i j}\left(f^{\prime}, f, t\right) & =\left|X_{i j}\left(f^{\prime}, f, t\right)\right|^{2} \\
& =\left|\int_{-\infty}^{\infty} H_{i j}\left(f^{\prime}, t^{\prime}\right) h\left(t^{\prime}-t\right) \exp \left(-2 \pi f t^{\prime}\right) d t^{\prime}\right|^{2}(11)
\end{aligned}
$$

where the Gaussian window is given by $h(t)=$ $1 /\left(\sqrt{\sigma_{w} \sqrt{\pi}}\right) \exp \left(-t^{2} /\left(2 \sigma_{w}^{2}\right)\right)$ is a real-valued, positive, and even function and has a normalized energy, i,e., $\int_{-\infty}^{\infty} h(t) d t=1$. In Eq. 11, the quantity $H_{i j}\left(f^{\prime}, t^{\prime}\right) h\left(t^{\prime}-t\right)$ is referred to as the short-time TVCTF [5, Eq. (2.3.1)], in which $t^{\prime}(t)$ is the running (local) time.

As described in the appendix, for short time intervals of duration $\delta t$, the TV path gain $c_{i j n}(t)$ corresponding to the $n$th moving scatterers $S_{n}^{M}(n=1,2, \ldots, M)$ can be assumed to be constant and are expressed as in Eeq. 24. In this case, and by following the same steps as in [4, Section IV], a closed-form approximate solution to the spectrogram can be determined as

$S_{i j}\left(f^{\prime}, f, t\right)=S_{i j}^{(a)}\left(f^{\prime}, f, t\right)+S_{i j}^{(c)}\left(f^{\prime}, f, t\right)$.

Here, the auto-term $S_{i j}^{(a)}\left(f^{\prime}, f, t\right)$ is expressed as

$$
\begin{aligned}
S_{i j}^{(a)}\left(f^{\prime}, f, t\right)= & \sum_{n=1}^{M} c_{i j n}^{2}(t) G\left(f,-\left(f_{c}+f^{\prime}\right) \dot{\tau}_{n}(t), \sigma_{n, 1}^{2}\left(f^{\prime}, t\right)\right) \\
& +c_{i j F}^{2}\left(f^{\prime}\right) G\left(f, 0, \frac{\sigma_{F}^{2}}{2}\right)
\end{aligned}
$$

while the cross-term $S_{i j}^{(c)}(f, t)$ is given in Eq. 10 (see the top of this page), where the operators $\operatorname{Re}\{\cdot\}$ and $(\cdot)^{*}$ return the real part and the complex conjugate of a complex 
number, respectively. In Eqs. 13 and $10, \sigma_{F}^{2}=1 /\left(2 \pi \sigma_{w}\right)^{2}$, $\sigma_{i j n, 1}^{2}\left(f^{\prime}, t\right)=\left[\sigma_{F}^{2}+\sigma_{w}^{2} k_{i j n}^{2}\left(f^{\prime}, t\right)\right] / 2$, and $\sigma_{i j n, 2}^{2}\left(f^{\prime}, t\right)=$ $\sigma_{F}^{2}-j k_{i j n}\left(f^{\prime}, t\right) /(2 \pi)$, in which $k_{i j n}\left(f^{\prime}, t\right)=-\left(f_{c}+\right.$ $\left.f^{\prime}\right) \ddot{\tau}(t)$, with $\ddot{\tau}(t)$ being the second-order time derivative of the TV delays $\tau_{i j n}(t)$.

By exploiting the results reported in [6, Eqs. (20) and (21)], the TV MDS $B_{i j}^{(1)}\left(f^{\prime}, t\right)$ and the TV DS $B_{i j}^{(2)}\left(f^{\prime}, t\right)$ can be determined from the spectrogram $S_{i j}\left(f^{\prime}, f, t\right)$ as

$B_{i j}^{(1)}\left(f^{\prime}, t\right)=\frac{\int_{-\infty}^{\infty} f S_{i j}\left(f^{\prime}, f, t\right) d f}{\int_{-\infty}^{\infty} S_{i j}\left(f^{\prime}, f, t\right) d f}$

and

$B_{i j}^{(2)}\left(f^{\prime}, t\right)=\sqrt{\frac{\int_{-\infty}^{\infty} f^{2} S_{i j}\left(f^{\prime}, f, t\right) d f}{\int_{-\infty}^{\infty} S_{i j}\left(f^{\prime}, f, t\right) d f}-\left(B_{i j}^{(1)}\left(f^{\prime}, t\right)\right)^{2}}$

respectively.

\section{Simulation results}

In this section, we explore the propagation scenario described in Section 4 by studying the effect of introducing the concept of TV path gains on the spectrogram $S_{i j}\left(f^{\prime}, f, t\right)$, the TV MDS $B_{i j}^{(1)}\left(f^{\prime}, t\right)$, and the TV DS $B_{i j}^{(2)}\left(f^{\prime}, t\right)$ of the TVCTF $H_{i j}\left(f^{\prime}, t\right)$ of the received RF signal.

As illustrated in Fig. 4, we consider a room of length $A=5 \mathrm{~m}$, width $B=10 \mathrm{~m}$, and height $H=2.4 \mathrm{~m}$. Here, the transmitter $T_{X}$ and the receiver $R_{X}$ consist of three transmit antennas $A_{j}^{T}(j=1,2,3)$ and three receive antennas $A_{i}^{R}(i=1,2,3)$, respectively. All omnidirectional antenna elements are mounted in pairs $\left(A_{i}^{T}, A_{i}^{R}\right)$ on the ceiling, where the elements $A_{i}^{T}$ and $A_{i}^{R}$ are separated by $0.5 \mathrm{~m}$. The fixed coordinates of the antennas $A_{1}^{T}, A_{2}^{T}, A_{3}^{T}$, $A_{1}^{R}, A_{2}^{R}$, and $A_{3}^{R}$ are $(0,-0.25,2.4),(2.5,-0.25,2.4),(5,-$ $0.25,2.4),(0,0.25,2.4),(2.5,0.25,2.4)$, and $(5,0.25,2.4)$, respectively.

A single walking person is modelled by 6 moving point scatterers, i.e., $M=6$. The scatterers represent the centre of mass of the major segments of the human body, namely, the head, ankles, wrists, and trunk (see Fig. 4). The selection of these specific body segments has been made in accordance with the analysis conducted in [16] and [19]. The authors of [16] and [19] studied the individual contribution of each segment to the overall human motor activities. The motion of the person is observed for a period
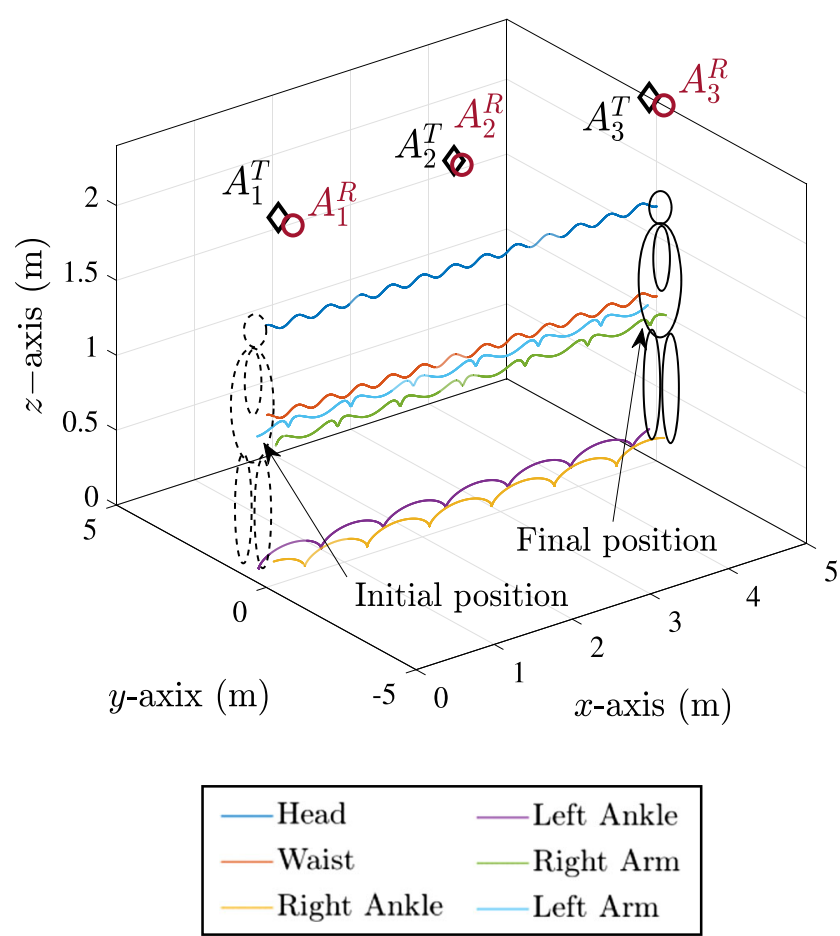

Fig. 4 3D simulation scenario with $M=6$ moving scatterers and a $3 \times 3$ MIMO system

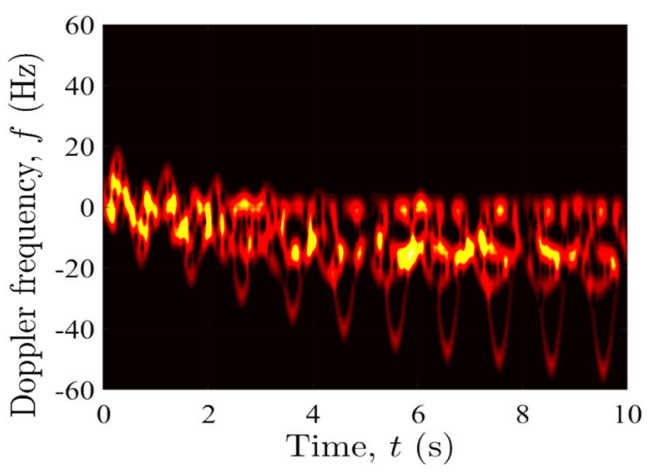

(a)

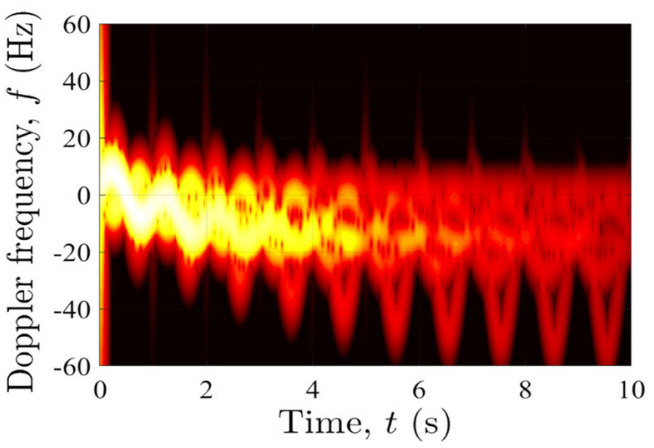

(b)

Fig. 5 Spectrogram $S_{11}(f, t)$ with a constant path gains and $\mathbf{b}$ TV path gains 
of $10 \mathrm{~s}$. The trajectories of the different moving scatterers (body segments) are simulated according to [3, Section V, Eqs. (24)-(29)]. As shown in Fig. 4, the person is first at one end of the room under antennas $A_{1}^{T}$ and $A_{1}^{R}$ and then moves to the other end of the room under antennas $A_{3}^{T}$ and $A_{3}^{R}$. The parameter $C$ and the path loss component $\gamma$ are set to 1000 and 1.6 , respectively. The center frequency $f_{c}$ was chosen to be $5.9 \mathrm{GHz}$. For clarity and without loss of generality, the figures presented are obtained for $f^{\prime}=0 \mathrm{~Hz}$. For comparison, the results for constant path gains and TV path gains are contrasted. For the case where the path gains of the moving scatterers are constant, the path gains are obtained by $c_{i j n}(t)=c_{i j n}=1$. The path gains of the fixed scatterers are given by $c_{k_{i j}}=\sqrt{2 / K_{i j}}$. The contribution of the fixed scatterers $S_{k_{i j}}$ is removed by applying a highpass filtering. For the remaining parameters as well as the scenario with constant path gains, we consider the same simulation parameters as described in [3, Section V].

Figures 5, 6, and 7 depict the spectrograms $S_{11}(0, f, t)$, $S_{22}(0, f, t)$, and $S_{33}(0, f, t)$, respectively. For comparison, we included here the spectrograms with constant path gains (Figs. 5a, 6a, and 7a) and the spectrograms with TV path gains (Figs. 5b, 6b, and 7b). From Figs. 5a, 6a, and 7a, it

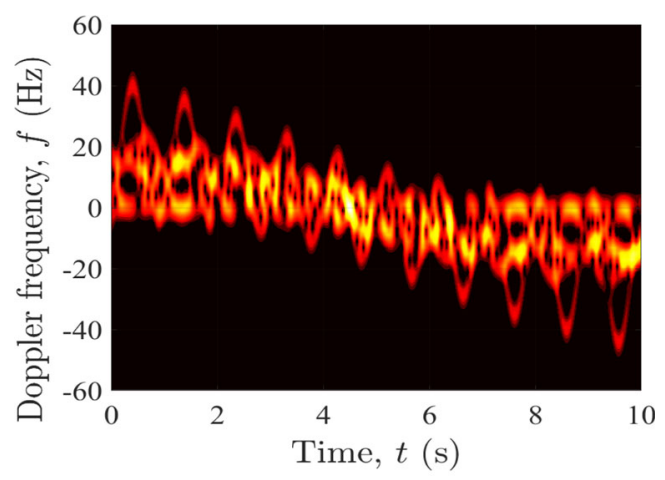

(a)

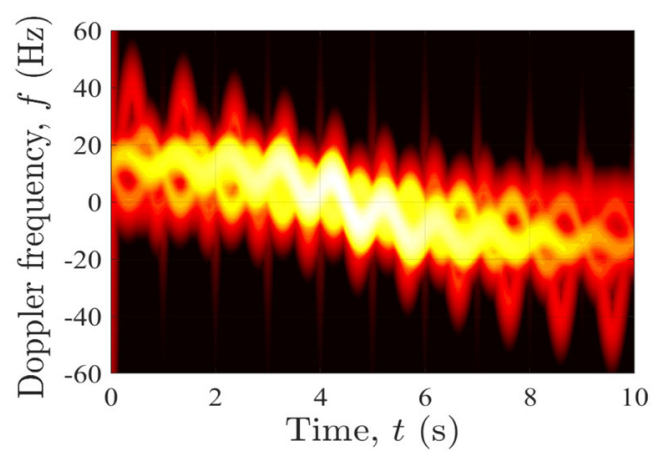

(b)

Fig. 6 Spectrogram $S_{22}(0, f, t)$ with a constant gains and $\mathbf{b} \mathrm{TV}$ path gains

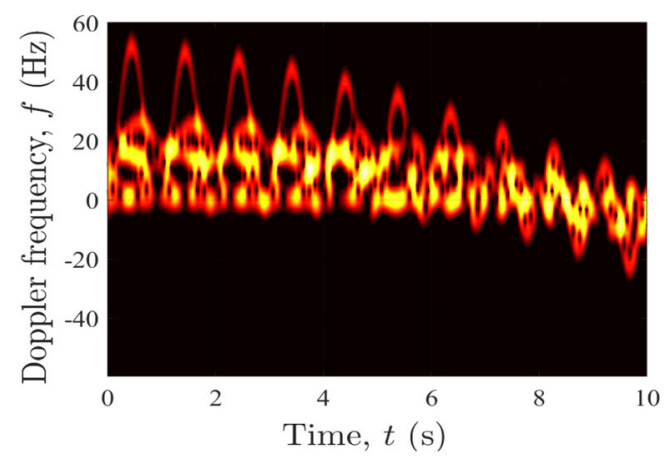

(a)

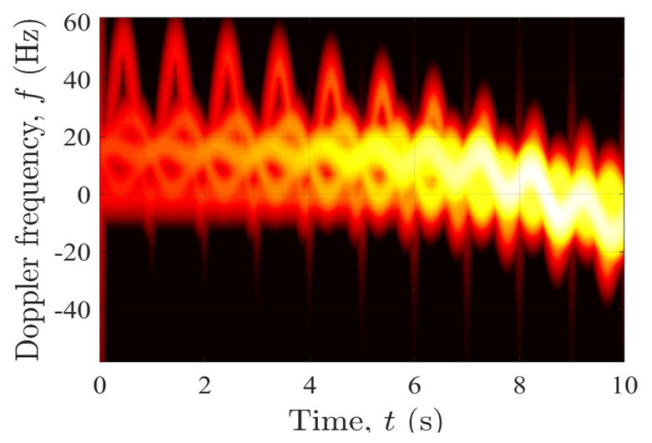

(b)

Fig. 7 Spectrogram $S_{33}(f, t)$ with a constant path gains and $\mathbf{b}$ TV path gains

can be observed that the average power of the spectrogram, which is proportional to the sum of the squared constant gains $\sum_{n=1}^{M} c_{i j n}^{2}$, remains constant along the observation time of $10 \mathrm{~s}$. The yellow parts of the spectrogram correspond to the superposition of the Gaussian function $G(a, b, c)$ (see Eqs. 13 and 10) and provide neither information about the motion of the body segments (moving point scatterers) nor the relative position of the person with respect to the transmit/receive antenna elements. This contrasts with Figs. 5b, 6b, and 7b, where the TV average power, which is proportional to the sum of the squared TV gains $\sum_{n=1}^{M} c_{i j n}^{2}(t)$, of the moving scatterers changes with time $t$. This stems from the fact that the TV path gains $c_{i j n}(t)$ are inversely proportional to the product of the TV distances $D_{1 n}^{T}(t)$ and $D_{1 n}^{R}(t)$ to the power of $\gamma / 2$. For example, in Fig. 5b, the person (modelled by a cluster of $M=6$ point scatterers) is moving away from the antenna pair $\left(A_{1}^{T}, A_{1}^{R}\right)$. Here, the TV distances $D_{1 n}^{T}(t)$ and $D_{1 n}^{R}(t)$ increase with respect to time $t$, which, in turn, results in decreasing TV average powers $c_{i j n}^{2}(t)$. In Fig. 6b, the person starts moving towards the antennas $A_{2}^{T}$ and $A_{2}^{R}$ and then moves away from this antennas pair. In this case, the TV power of the spectrogram increases until the person is located below $\left(A_{2}^{T}, A_{2}^{R}\right.$ ) (at approximately $t=4.5 \mathrm{~s}$ ) and then begins to decrease until the person reaches its final position. Finally, 
Fig. $7 \mathrm{~b}$ depicts the spectrogram $S_{33}(0, f, t)$ of the TVCTF $H_{33}(0, t)$, where the person walks towards the antennas $A_{3}^{T}$ and $A_{3}^{R}$.

In Figs. 5a, 6a, and 7a, the constant power of the spectrograms can be physically interpreted by the fact that the range of the RF system is infinite. This means that the motion of the person can be observed regardless of how far or how close it is positioned with respect to the transmit and receive antennas. In reality, this is not the case, especially for indoor wireless communications. By introducing instantaneous path gains for the moving scatterers $S_{n}^{M}(n=1,2, \ldots, M)$ (see Figs. $5 \mathrm{~b}, 6 \mathrm{~b}$, and $\left.7 \mathrm{~b}\right)$, the TV behavior of the power of the spectrograms can be

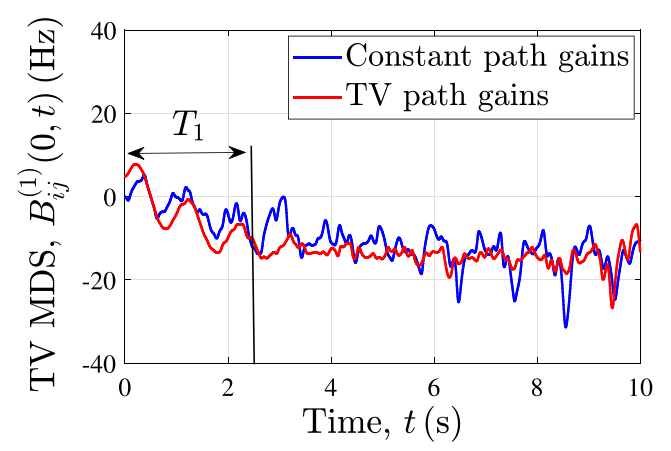

(a)

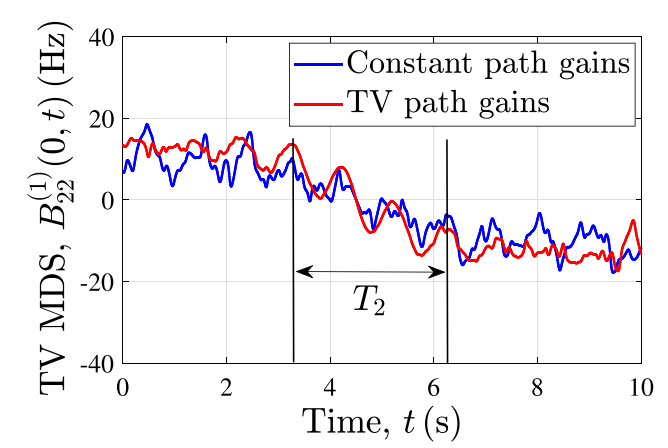

(b)

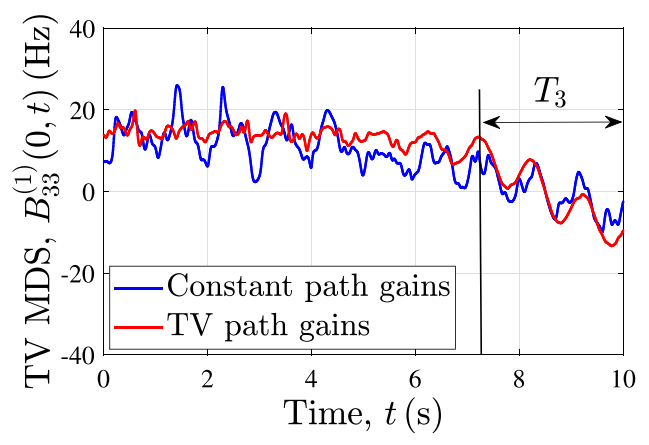

(c)

Fig. 8 TV mean Doppler shift (MDS) $B_{i j}^{(1)}(0, t)$ observed between the antennas $\mathbf{a} A_{1}^{T}$ and $A_{1}^{R}$, b $A_{2}^{T}$ and $A_{2}^{R}$, and $\mathbf{c} A_{3}^{T}$ and $A_{3}^{R}$ explained by the fact that by moving away from (towards) the $T_{X}$ antennas and the $R_{X}$ antennas, the person is walking out of (into) the range of the RF system. This is the behavior observed in practice, which confirms the use of TV path gains to describe the motion of the different human body segments.

To further investigate the effect of introducing TV path gains to describe the moving scatterers $S_{n}, n=1,2, \ldots, M$, in the expression of the TVCTF $H_{i j}\left(f^{\prime}, t\right)$, on the Doppler characteristics of the fading channel model, we study the TV MDS $B_{i j}^{(1)}(0, t)$ and TV DS $B_{i j}^{(2)}(0, t)$. Let us denote by $T_{1}, T_{2}$, and $T_{3}$ the time intervals during which the person in moving close to the antenna elements $\left(A_{1}^{T}, A_{1}^{R}\right),\left(A_{2}^{T}, A_{2}^{R}\right)$,

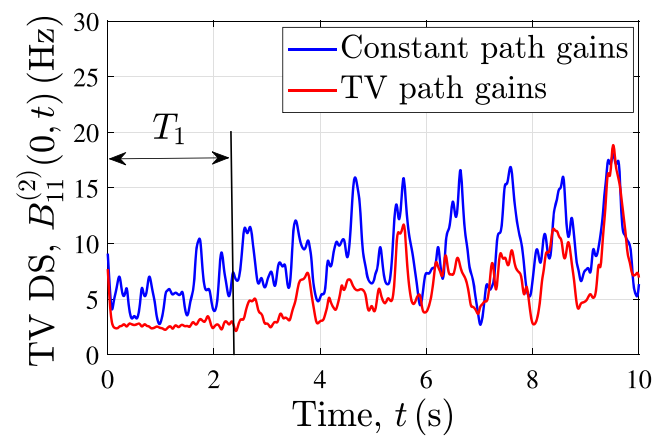

(a)

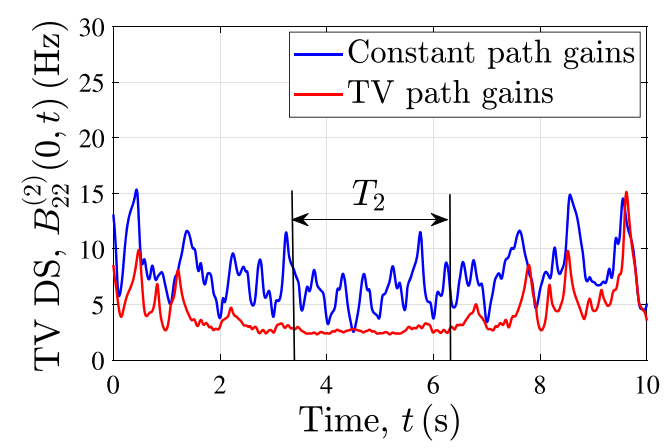

(b)

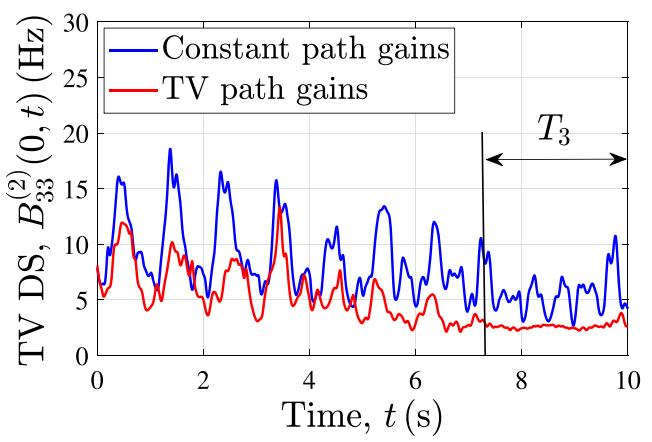

(c)

Fig. 9 TV Doppler spread (DS) $B_{i j}^{(2)}(0, t)$ observed between the antennas $\mathbf{a} A_{1}^{T}$ and $A_{1}^{R}, \mathbf{b} A_{2}^{T}$ and $A_{2}^{R}$, and $\mathbf{c} A_{3}^{T}$ and $A_{3}^{R}$ 
and $\left(A_{3}^{T}, A_{3}^{R}\right)$, respectively. We present in Fig. 9a, b, and c a comparison of the TV MDSs $B_{i j}^{(1)}(0, t)$ using constant path gains and TV path gains for the different pairs of antennas $\left(A_{1}^{T}, A_{1}^{R}\right),\left(A_{2}^{T}, A_{2}^{R}\right)$, and $\left(A_{3}^{T}, A_{3}^{R}\right)$, respectively. The TV MDSs $B_{i j}^{(1)}(0, t)$ were numerically computed according to Eq. 14. As can be seen, the introduction of TV path gains only slightly influences the trend of the TV MDS. However, it strongly affects the values of $B_{i j}^{(1)}(0, t)$, especially when the person is not in close proximity to the antenna pairs, i.e., outside the intervals $T_{1}$, $T_{2}$, and $T_{3}$. The TV DSs $B_{i j}^{(2)}(0, t)$ were computed from the spectrograms $S_{i j}(0, f, t)$ utilizing (15). It is shown in Fig. 8a, b, and c that the consideration of the TV path gains $c_{i j}(t)$ influences both the trend and the values of the TV DSs $B_{i j}^{(2)}(0, t)$. We also observe that the TV DSs $B_{1}^{(2)}(0, t), B_{22}^{(2)}(0, t)$, and $B_{33}^{(2)}(0, t)$ remain almost constant during the time durations $T_{1}, T_{2}$, and $T_{3}$, receptively. This means that the Doppler frequencies do not deviate much from the $\operatorname{MDS} B_{i j}^{(1)}(0, t)$. In other words, during $T_{1}, T_{2}$, and $T_{3}$ (see Fig. 8a, b, and c), the moving scatterers (segments) modelling the human body can be replaced by a single moving scatterer, whose corresponding Doppler frequency can be approximated by the TV $\left.\operatorname{MDS} B_{11}^{(1)}(0, t)\right|_{t \in T_{1}}$, $\left.B_{22}^{(1)}(0, t)\right|_{t \in T_{2}}$, and $\left.B_{33}^{(1)}(0, t)\right|_{t \in T_{3}}$ (see Fig. 9). This can be explained by the fact that as the person gets closer to the antennas, the main body segment describing the motion of the person is the head. All other body parts are obstructed because the antennas are attached to the ceiling.

\section{Conclusion}

This paper introduces a new 3D non-stationary indoor MIMO channel model, in which a single person is moving. The different segments making up the human body are modelled by a cluster of synchronized moving point scatterers. Taking advantage from the fact that an electric current is induced in the human body when it is exposed to low-frequency electromagnetic fields, we assume that the human body segments (moving scatterers) play the role of moving relays, which redirect the transmit radio signal. We present an expression for the TVCTF of the received RF signal with TV path gains and TV path delays. The TV path gain of each multipath component resulting from the moving person (moving scatterers) is modelled by means of the free-space path loss model. The impact of the TV distances between the moving person (scatterer) and the transmit and receive antenna elements on the spectrogram, the TV MDS, and the TV DS of the TVCTF is analyzed. It is concluded that this new expression for the TV path gains has two main advantages. First, it allows to detect the relative position of the person with respect to the transmit and receive antennas. Second, it gives insight into the range of the considered RF system. In fact, the closer the person is to the antennas, the higher is the power of the spectrogram. Moreover, when the person is out of range of the transmit/receive antennas (far away), the motion of the person cannot be clearly visualized in the spectrogram. The obtained analytical results have been illustrated by computer simulations.

$$
\begin{aligned}
c_{i j n}(t) \approx & \frac{a_{n} C}{\left[D_{j n}^{T}\left(t_{0}\right) D_{i n}^{R}\left(t_{0}\right)\right]^{\gamma}}\left\{1-\frac{\gamma\left(t-t_{0}\right)}{2}\left[\left(x_{n}(t) x_{n}^{\prime}\left(t_{0}\right)+y_{n}(t) y_{n}^{\prime}\left(t_{0}\right)+z_{n}(t) z_{n}^{\prime}\left(t_{0}\right)\right)\left(\left(D_{j n}^{T}\left(t_{0}\right)\right)^{-2}+\left(D_{i n}^{R}\left(t_{0}\right)\right)^{-2}\right)\right.\right. \\
& \left.\left.-\left(x_{n}^{\prime}\left(t_{0}\right)+y_{n}^{\prime}\left(t_{0}\right)+y_{n}^{\prime}\left(t_{0}\right)\right)\left(\frac{x_{j}^{T}+y_{j}^{T}+z_{j}^{T}}{\left(D_{j n}^{T}\left(t_{0}\right)\right)^{2}}+\frac{x_{i}^{R}+y_{i}^{R}+z_{i}^{R}}{\left(D_{i n}^{R}\left(t_{0}\right)\right)^{2}}\right)\right]\right\} .
\end{aligned}
$$

\section{Appendix}

This appendix explains the mathematical manipulations leading to the approximate solutions to the TV path gains $c_{i j n}(t)$ in (6). To do this, we apply a Taylor series expansion to the TV displacements $x_{n}(t), y_{n}(t)$, and $z_{n}(t)$ of the $n$th moving scatterer (modelling the $n$th body segment). Since the objective is to justify the assumption that the TV path gains $c_{i j n}(t)$ can be assumed to be constant for short periods of time, the following mathematical calculations will be conducted using a first-order Taylor expansion.
In other words, for short time intervals centered at the time instant $t_{0}$ and of duration $\delta t$, the TV positions $x_{n}(t)$, $y_{n}(t)$, and $z_{n}(t)$ can be written as

$$
\begin{aligned}
& x_{n}(t) \approx x_{n}\left(t_{0}\right)+\left(t-t_{0}\right) x_{n}^{\prime}\left(t_{0}\right) \\
& y_{n}(t) \approx y_{n}\left(t_{0}\right)+\left(t-t_{0}\right) y_{n}^{\prime}\left(t_{0}\right)
\end{aligned}
$$

and

$z_{n}(t) \approx z_{n}\left(t_{0}\right)+\left(t-t_{0}\right) z_{n}^{\prime}\left(t_{0}\right)$ 
for $t \in\left[t_{0}-\delta t / 2, t_{0}+\delta t / 2\right]$, respectively. In Eqs. 17-19, $u^{\prime}(t)$ refers to the time derivative of the function $u(t)$. Then, the TV distances $D_{j n}^{T}(t)$ and $D_{i n}^{R}(t)$ can be approximated by

$$
\begin{gathered}
D_{j n}^{T}(t) \approx\left[\left(D_{j n}^{T}\left(t_{0}\right)\right)^{2}+\left(t-t_{0}\right)\left(x_{n}(t) x_{n}^{\prime}\left(t_{0}\right)+y_{n}(t) y_{n}^{\prime}\left(t_{0}\right)\right.\right. \\
\left.\left.\quad+z_{n}(t) z_{n}^{\prime}\left(t_{0}\right)-x_{n}^{\prime}\left(t_{0}\right) x_{j}^{T}-y_{n}^{\prime}\left(t_{0}\right) y_{j}^{T}-y_{n}^{\prime}\left(t_{0}\right) y_{j}^{T}\right)\right]^{1 / 2}
\end{gathered}
$$

and

$$
\begin{gathered}
D_{i n}^{R}(t) \approx\left[\left(D_{i n}^{R}\left(t_{0}\right)\right)^{2}+\left(t-t_{0}\right)\left(x_{n}(t) x_{n}^{\prime}\left(t_{0}\right)+y_{n}(t) y_{n}^{\prime}\left(t_{0}\right)\right.\right. \\
\left.\left.+z_{n}(t) z_{n}^{\prime}\left(t_{0}\right)-x_{n}^{\prime}\left(t_{0}\right) x_{i}^{R}-y_{n}^{\prime}\left(t_{0}\right) y_{i}^{R}-y_{n}^{\prime}\left(t_{0}\right) y_{i}^{R}\right)\right]^{1 / 2}
\end{gathered}
$$

for $t \in\left[t_{0}-\delta t / 2, t_{0}+\delta t / 2\right]$, respectively. By placing the transmit antennas $A_{j}^{T}$ and receive antennas $A_{i}^{R}$ in such way that the distances $D_{j n}^{T}\left(t_{0}\right)$ and $D_{i n}^{R}\left(t_{0}\right)$ are larger than 1 (e.g., on the ceiling or in the corners of the room, which ensures maximum coverage) and using $(1+x)^{\alpha} \approx 1+\alpha x$, the TV quantities $\left(D_{j n}^{T}(t)\right)^{-\gamma}$ and $\left(D_{i n}^{R}(t)\right)^{-\gamma}$ can be obtained as

$$
\begin{aligned}
\left(D_{j n}^{T}(t)\right)^{-\gamma} \approx & \left(D_{j n}^{T}\left(t_{0}\right)\right)^{-\gamma}\left[1-\frac{\gamma\left(t-t_{0}\right)}{2\left(D_{j n}^{T}\left(t_{0}\right)\right)^{2}}\left(x_{n}(t) x_{n}^{\prime}\left(t_{0}\right)\right.\right. \\
& +y_{n}(t) y_{n}^{\prime}\left(t_{0}\right)+z_{n}(t) z_{n}^{\prime}\left(t_{0}\right)-x_{n}^{\prime}\left(t_{0}\right) x_{j}^{T} \\
& \left.\left.-y_{n}^{\prime}\left(t_{0}\right) y_{j}^{T}-y_{n}^{\prime}\left(t_{0}\right) y_{j}^{T}\right)\right]
\end{aligned}
$$

and

$$
\begin{aligned}
\left(D_{i n}^{R}(t)\right)^{-\gamma} \approx & \left(D_{i n}^{R}\left(t_{0}\right)\right)^{-\gamma}\left[1-\frac{\gamma\left(t-t_{0}\right)}{2\left(D_{i n}^{R}\left(t_{0}\right)\right)^{2}}\left(x_{n}(t) x_{n}^{\prime}\left(t_{0}\right)\right.\right. \\
& +y_{n}(t) y_{n}^{\prime}\left(t_{0}\right)+z_{n}(t) z_{n}^{\prime}\left(t_{0}\right)-x_{n}^{\prime}\left(t_{0}\right) x_{i}^{R} \\
& \left.\left.-y_{n}^{\prime}\left(t_{0}\right) y_{i}^{R}-y_{n}^{\prime}\left(t_{0}\right) y_{i}^{R}\right)\right]
\end{aligned}
$$

for $t \in\left[t_{0}-\delta t / 2, t_{0}-\delta t / 2\right]$, respectively. Inserting (22) and (23) in (6) yields the first-order approximate solution to the TV path gains $c_{i j n}(t)$ in Eq. 16 [see the top of this page], for time instance $t$ in the interval $\left[t_{0}-\delta t / 2, t_{0}-\delta t / 2\right]$.

Profiting from the facts that $\left|t-t_{0}\right|<\delta t, D_{j n}^{R}\left(t_{0}\right) \gg 1$, $D_{i n}^{T}\left(t_{0}\right) \gg 1$, and $\gamma / 2 \leq 1$, the second term of Eq. 16 can be neglected, which in turn results in the following expression for the TV path gains $c_{i j n}(t)$ for short time intervals of duration $\delta t$

$c_{i j n}(t) \approx a_{n} C\left(D_{j n}^{T}\left(t_{0}\right) D_{i n}^{R}\left(t_{0}\right)\right)^{-\gamma}$.

For simplicity, we have considered a first-order Taylor expansion for the TV displacements to show that the TV path gains $c_{i j n}(t)$ can be assumed to be constant for short time intervals of duration $\delta t$. In case of the higher-order Taylor expansion of $x_{n}(t), y_{n}(t)$, and $z_{n}(t)$, it can be similarly argued that higher-order terms can be neglected in the approximation of the TV path gains $c_{i j n}(t)$.

Funding Open access funding provided by University of Agder. This work was carried out within the scope of the WiCare Project funded by the Research Council of Norway under grant number 261895/F20.

Open Access This article is licensed under a Creative Commons Attribution 4.0 International License, which permits use, sharing, adaptation, distribution and reproduction in any medium or format, as long as you give appropriate credit to the original author(s) and the source, provide a link to the Creative Commons licence, and indicate if changes were made. The images or other third party material in this article are included in the article's Creative Commons licence, unless indicated otherwise in a credit line to the material. If material is not included in the article's Creative Commons licence and your intended use is not permitted by statutory regulation or exceeds the permitted use, you will need to obtain permission directly from the copyright holder. To view a copy of this licence, visit http://creativecommons. org/licenses/by/4.0/.

\section{References}

1. Federal public service: Interaction between radiation and the human body (2019). Available: https://www.health.belgium.be/ en/interaction-between-radiation-and-human-body-article

2. United nations: Transforming our world: the 2030 agenda for sustainable development (2015). Available: https://www.un.org/ $\mathrm{ga} /$ search/view_doc.asp?symbol=A/RES/70/1\&Lang=E

3. Abdelgawwad A, Pätzold M (2019) A 3D non-stationary cluster channel model for human activity recognition. In: IEEE 89Th veh. Technol. Conf. (VTC'19-spring), Kuala Lumpur, pp 1-7

4. Abdelgawwad M, Pätzold M (2017) On the influence of walking people on the Doppler spectral characteristics of indoor channels. In: IEEE 28Th annual int. Symp. on personal, indoor, and mobile radio commun. (PIMRC'17), Montreal, pp 1-7

5. Boashash B (2015) Time-frequency signal analysis and processing: a comprehensive reference. Academic Press

6. Borhani A, Pätzold M. (2018) A non-stationary channel model for the development of non-wearable radio fall detection systems. IEEE Trans Wirel Commun 17(11):7718-7730

7. Chen Y, Ou R, Li Z, Wu K (2020) WiFace: facial expression recognition using Wi-Fi signals. IEEE Trans Mob Comput:1-15

8. Council NR et al (1993) Effects of electromagnetic fields on organs and tissues. In: Assessment of the possible health effects of ground wave emergency network. National Academies Press, US

9. Council NR et al (1993) Human laboratory and clinical evidence of effects of electromagnetic fields. In: Assessment of the possible health effects of ground wave emergency network. National Academies Press, US

10. Fischinger D et al (2014) Hobbit, a care robot supporting independent living at home: first prototype and lessons learned. Robot Auton Syst 75:60-78

11. Friis HT (1946) A note on a simple transmission formula. IEEE Proc IRE 34(5):254-256

12. Gallo P, Mangione S (2015) RSS-eye: Human-assisted indoor localization without radio maps. In: IEEE Int. Conf. on commun. (ICC'15), London, pp 1553-1558

13. Hicheri R, Abdelgawwad A, Pätzold M (2020) A new nonstationary $3 \mathrm{D}$ channel model with time-variant path gains for indoor human activity recognition. In: $2 \mathrm{Nd}$ int. Conf. on telecom. and comp. Technol. (ICTCT'20), hammamet

14. Hussain Z, Sheng M, Zhang WE (2019) Different approaches for human activity recognition: a survey. arXiv:1906.05074 
15. Jalal A, Uddin MZ, Kim TS (2012) Depth video-based human activity recognition system using translation and scaling invariant features for life logging at smart home. IEEE Trans Consum Electron 58(3):863-871

16. Jobanputra C, Bavishi J, Doshi N (2019) Human activity recognition: a survey. Procedia Comput Sci 155:698-703

17. Khurana R, Singh Kushwaha AK (2018) A deep survey on human activity recognition in video surveillance. In: Int. Conf. on research in intelligent and computing in engineering (RICE'18), San Salvador, pp 1-5

18. Kotterman WAT, Pedersen GF, Olesen K (2004) Diversity properties of multi-antenna small handheld terminals. EURASIP J Appl Signal Proc 9:1340-1353

19. Lenzi D, Cappello A, Chiari L (Sep. 2003) Influence of body segment parameters and modeling assumptions on the estimate of center of mass trajectory. J Biomech 36(9):1335-1341

20. Li Y, Jiang T, Ding X, Wang Y (2020) Location-free CSI based activity recognition with angle difference of arrival. In: IEEE Wireless commun. and networking conf. (WCNC'20), Seoul, pp 1-6

21. Liu J, Liu H, Chen Y, Wang Y, Wang C (Aug. 2019) Wireless sensing for human activity: a survey. IEEE Commun Surveys Tuts

22. Mahmoudinasab H, Sanie-Jahromi F, Saadat M (2016) Effects of extremely low-frequency electromagnetic field on expression levels of some antioxidant genes in human MCF-7 cells. Mol Biol Res Commun 5(2):77-85

23. Makki A, Siddig A, Saad M, Cavallaro JR, Bleakley CJ (2016) Indoor localization using 802.11 time differences of arrival. IEEE Trans. Instrum Meas 65(3):614-623

24. Muramatsu S, Kasano E, Motohashi Y, Sato-Shimokawara E, Chan R. Y, Yamaguchi T (2019) Estimating indoor behavior using sensor fusion with BLE beacons and wearable devices for information presentation. In: 16Th int. Conf. on ubiquitous robots (UR'19), Jeju, pp 236-239

25. Nakagawa E, Moriya K, Suwa H, Fujimoto M, Arakawa Y, Yasumoto K (2017) Toward real-time in-home activity recognition using indoor positioning sensor and power meters. In: IEEE Int. Conf. on pervasive computing and commun. Workshops (percom workshops'17), Kona, pp 539-544

26. Organick EI (1966) Some processors also offer the library function called ATAN2 a function of two arguments (opposite and adjacent). In: A FORTRAN IV primer. Addison-wesley, pp 42

27. Pätzold M, Gutierrez CA (2018) Modelling of non-WSSUS channels with time-variant Doppler and delay characteristics. In: IEEE 7Th int. Conf. on commun. and electron. (ICCE'18), Hue City, pp 1-6

28. Pham M, Yang D, Sheng W (2019) A sensor fusion approach to indoor human localization based on environmental and wearable sensors. IEEE Trans Autom Sci Eng 16(1):339-350

29. Phillips C, Sicker D, Grunwald D (2013) A survey of wireless path loss prediction and coverage mapping methods. IEEE Commun. Surv Tuts 15(1):255-270

30. Seneviratne $S$ et al (2017) A survey of wearable devices and challenges. IEEE Commun. Surv Tuts 19(4):2573-2620

31. Shoaib M, Elbrandt T, Dragon R, Ostermann J (2010) Altcare: Safe living for elderly people. In: 4Th IEEE int. Conf. on pervasive computing technol. for healthcare (pervasivehealth'10, Munich, pp $1-4$

32. Sun YL, Xu YB (2013) Error estimation method for matrix correlation-based Wi-Fi indoor localization. KSII Trans Internet Inf Syst 7(11):2657-2675

33. Yassin A et al (2017) Recent advances in indoor localization: A survey on theoretical approaches and applications. IEEE Commun Surv Tuts 19(2):1327-1346

34. Yoshida-Intern S (2007) A global report on falls prevention epidemiology of falls. World Health Organization, Geneva

Publisher's note Springer Nature remains neutral with regard to jurisdictional claims in published maps and institutional affiliations. 